\title{
BCG vaccine and COVID-19: implications for infection prophylaxis and cancer immunotherapy
}

\author{
Madhuri Koti, ${ }^{1,2}$ Alvaro Morales, ${ }^{2}$ Charles H Graham, ${ }^{1,2}$ David Robert Siemens ${ }^{1,2}$
}

To cite: Koti M, Morales A, Graham $\mathrm{CH}$, et al. $\mathrm{BCG}$ vaccine and COVID-19: implications for infection prophylaxis and cancer immunotherapy. Journal for ImmunoTherapy of Cancer 2020;8:e001119. doi:10.1136/ jitc-2020-001119

Accepted 20 June 2020

Check for updates

(C) Author(s) (or their employer(s)) 2020. Re-use permitted under CC BY-NC. No commercial re-use. See rights and permissions. Published by BMJ.

${ }^{1}$ Biomedical and Molecular Sciences, Queen's University, Kingston, Ontario, Canada

${ }^{2}$ Department of Urology, Queen's University, Kingston, Ontario, Canada

Correspondence to

Dr Madhuri Koti;

kotim@queensu.ca

\begin{abstract}
The COVID-19 pandemic has killed over 400000 people globally. Ecological evidence indicates that countries with national universal BCG vaccination programs for tuberculosis (TB) prevention have a much lower incidence of severe COVID-19 and mortality compared with those that do not have such programs. BCG is a century old vaccine used for TB prevention via infant/childhood vaccination in lowto middle-income countries with high infection prevalence rate and is known to reduce allcause neonatal mortality. BCG remains the standard immunotherapy treatment for patients with high-risk non-muscle invasive bladder cancer globally for more than 44 years. Several trials are, therefore, investigating BCG as a prophylactic against COVID-19 in healthcare workers and the elderly. In this commentary, we discuss the potential mechanisms that may underlie BCG associated heterologous protection with a focus on tertiary lymphoid structure (TLS) organogenesis. Given the significance of TLSs in mucosal immunity, their association with positive prognosis and response to immune checkpoint blockade with a critical role of Type I interferon (IFN-1) in inducing these, we also discuss potentiating TLS formation as a promising approach to enhance anti-tumor immunity. We propose that lessons learned from BCG immunotherapy success could be applied to not only augment such microbe-based therapeutics but also lead to similar adjunctive IFN-1 activating approaches to improve response to immune checkpoint blockade therapy in cancer.
\end{abstract}

The observation that microbes could curb cancer growth was made over 4000 years ago when incisions on tumors led to infection mediated spontaneous regression. In modern medicine, the therapeutic potential of microbes, or their components, against cancer was first demonstrated by William Coley in 1891. Although Coley's toxins did not come into wide clinical practice due to inconsistencies in the type of cancers treated, routes of administration and variable outcomes from this clinical experience established the proof-of-concept that stimulating the immune system using microbes or their components can be an adjunctive treatment to surgery and cytotoxic therapy. Although this was followed by sporadic successes in other solid tumors, a major success pioneering the field of cancer immunotherapy was introduction of intravesical administration of BCG for the treatment of non-muscle invasive bladder cancer (NMIBC). ${ }^{1}$ This was one of the very few initial testaments of benefits from weakened or modified microbes and their specific molecular patterns for antitumor immune sensitization. Despite ongoing global shortages, BCG immunotherapy remains the goldstandard treatment for NMIBC. Over the last 100 years, BCG vaccine has been the only tuberculosis (TB) preventative strategy and is quite eloquently described as 'the vaccine that keeps on giving'. ${ }^{2}$ Infant/childhood BCG vaccination via intradermal administration continues to be practiced in several countries to prevent $\mathrm{TB}^{2}{ }^{2}$ An intriguing association between BCG vaccination and incidence from the ongoing COVID-19 is a recent stirring although a controversial observation. Countries with national vaccination programs (eg, India, Korea, Japan) for TB prevention, using BCG vaccine, showed a much lower morbidity and mortality from severe COVID-19 compared with those (eg, Italy, USA, UK) that do not have such policies. ${ }^{3}$ Although this observation was not confirmed by others, ${ }^{4}$ an immediate response to this ecological finding was initiation of COVID-19 clinical trials evaluating BCG (https://clinicaltrials.gov/ ct $2 /$ results? cond=BCG+and+COVID-19) and its recombinant form VPM1002 (https:// clinicaltrials.gov/NCT04387409), to protect healthcare workers and the elderly.

BCG remains as one of the best-known vaccines associated with beneficial and heterologous protection from other diseases. The 'pathogen agnostic' effects of BCG span from reducing sepsis associated mortality, ${ }^{2}$ protection against other forms of TB, potentiating antiviral (Respiratory syncitial virus, yellow fever caused by an RNA virus) immune responses and most importantly reducing 
cancer recurrence and progression. ${ }^{5}$ This non-specific effect of BCG is described to be at least partially mediated via innate immune memory/trained immunity through microbe-induced epigenetic changes in cells of the innate and adaptive immune systems. ${ }^{5}$ While trial outcomes from BCG vaccination induced protection of healthcare workers from COVID-19 are awaited to further support this interesting and promising association, several questions arise: (1) Is this protective effect age- and sexdependent? (2) How long does the protection last? (3) Can BCG synergize with currently approved immunomodulatory agents? (4) Given the variability in immune cell composition across tissues, is the effect location/ tissue type dependent? (5) Does the dose, route, duration, number and frequency of administration weigh on the effect? (6) Does the host genetics influence response? (7) Will the strain of BCG vaccine induce a variable response? (8) Will lung associated comorbidities cause a deleterious effect due to a chronic inflammatory state and most importantly (9) How could these investigations impact NMIBC treatment?

While revisiting the parallels of antiviral and anticancer immunity, interferons (IFN) undoubtedly lie at a major intersection between these two pathologies. Viral infection antagonizes cytosolic innate sensing pathways such as the Retinoic acid-inducible gene (RIG-1) and cyclic guanosine monophosphate-adenosine monophosphate synthase (cGAS)-STimulator of INterferon Genes (STING) pathways to evade protective IFN response. Mimicking such viral immune evasion mechanisms, cancer cells often adapt to an impaired IFN-1 signaling and thus modulate the associated microenvironment toward a suppressive phenotype conducive to their growth and metastasis. Both type I and II IFN (IFN- $\alpha / \beta$ and IFN- $\gamma$, respectively) are critical to these effects and, therefore, effort has focused on their direct or indirect activation via vaccination and/or therapeutic introduction in order to induce or magnify a protective IFN response.

This brings us to the question; How does BCG impart its non-specific protective effect against viral infections or its anticancer effect and are they IFN dependent? Studies from Netea and colleagues have extensively investigated and explained this concept and role of innate immune memory within macrophages. ${ }^{5}$ Yao et al further demonstrated that at mucosal sites, $\mathrm{CD} 8^{+} \mathrm{T}$ cell help was indispensable for priming of such memory within the lung resident alveolar macrophages. ${ }^{6}$ Subsequent downstream effects extrinsic to macrophages, such as chemokine mediated immune cell recruitment to the site of infection/inflammation, further activation of the adaptive immune responses and the influence of tissue specific immune microenvironment are some key factors to the overall favorable outcomes that depend on the initial magnitude of macrophage priming. In our effort to further gain a deeper understanding of BCG induced protective effects and enhance them, we must consider the unique pathogenesis of Mycobacterial infection and host responses towards controlling its spread. One such unique feature that is seen across the spectrum of various TB states, is granuloma formation. ${ }^{7}$ Granuloma formation is considered to be a hallmark of Mycobacterial infection. Mycobacterium tuberculosis (MTB) induced granuloma and is constituted by an aggregate of macrophages and lymphocytes and develops as a result of host immune responses to contain the bacterial spread. Interestingly, TB associated/pathogenic Mycobacteria-induced protective, chronic granuloma also exhibits remarkable similarities to an ectopic/tertiary lymphoid structure (TLS), a predominant feature of mucosa associated lymphoid tissue containing innate and adaptive immune cells with similar architecture as seen in a canonical germinal center in secondary lymphoid organs. ${ }^{7}$ Indeed, IFN-1 signaling and downstream immune cell chemotaxis to the site of infection, are central to the formation of these TLSs and maturation of B cells including antibody class switching and affinity maturation within these. ${ }^{8}$

Following birth, TLSs develop at mucosal sites (eg, respiratory, gut and urogenital tract). TLSs also form as a result of exposure to normal commensal and pathogenic microbes and chronic inflammation (eg, chronic obstructive pulmonary disease; COPD and cancer) and generally provide heterologous humoral immune protection. ${ }^{8}$ Higher frequency of these TLSs, also known as induced bronchus associated lymphoid tissues (iBALTs) in the respiratory mucosa, is protective against respiratory infections such as Influenza A virus, severe acute respiratory syndrome coronavirus $(S A R S-C o V)$ and MTB ${ }^{8}$ The proximity of vaccine-induced iBALTs to respiratory pathogens leads to rapid B-cell maturation, antibody production and $\mathrm{T}$ cell priming. Mucosal delivery of BCG (either lung or bladder) also leads to delayed granuloma/iBALT formation as well as B cell activation, reminiscent of pathogenic Mycobacteria-associated granuloma. In contrast, pathogenic iBALTs also develop in autoimmune diseases and in respiratory pathologies such as asthma, smoking, hypertension, COPD. Irrespective of the initial insult/challenge, IFN-1 is critical to TLS organogenesis. ${ }^{8}$

Similar to mucosal vaccination associated granuloma formation, in the case of NMIBC, BCG delivered into the bladder lumen/mucosa also leads to granuloma formation. ${ }^{910}$ To compensate for lack of response to BCG given its inability to induce IFN-1 activation, exogenously delivered recombinant IFN- $\alpha$ has been extensively evaluated in this cancer. ${ }^{10}$ Adenoviral vector expressing IFN- $\alpha 2 b$ is also currently in phase III trials (https://clinicaltrials.gov/ NCT02773849). Over the past few years, several studies have been conducted to identify factors underlying reduced efficacy of BCG to activate an IFN-1 response.

BCG is derived via attenuation of Mycobacterium bovis. The loss of early secretory antigenic target secretion system-1 (ESX-1), one of the immunodominant antigens, as a result of attenuation for vaccine preparation, impairs the ability of BCG to activate the cGAS-STING/ IRF3, RIG/MAVS/IRF7 cytosolic nucleic acid sensing pathways. ${ }^{11}$ As such, there is minimal IFN- $\beta$ production, while some protection is imparted through cytokine 


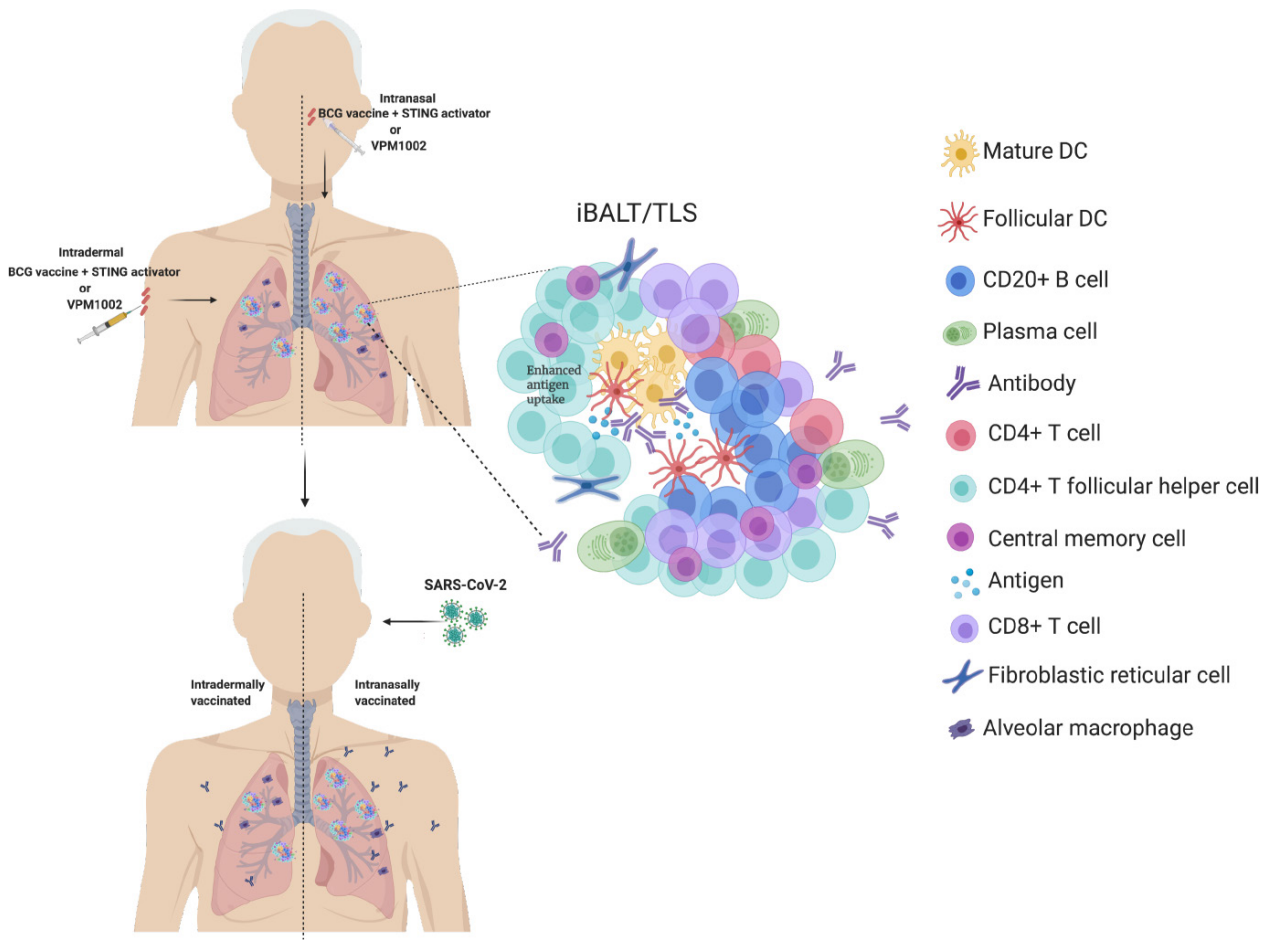

Figure 1 Hypothetical diagram showing enhanced protection by intranasally delivered BCG (in combination with a sting pathway activator) or with recombinant BCG (VPM1002), against SARS-CoV2 viral infection. iBALT, induced bronchus associated lymphoid tissue; STING, STimulator of INterferon Genes; TLS, tertiary lymphoid structure; DC, Dendritic cell; SARSCoV2, Severe acute respiratory syndrome-coronavirus 2.

induction and downstream immunological effects via NLRP3 inflammasome activation within macrophages, dendritic cells and epithelial cells. ${ }^{11}$ To compensate for this IFN-1 activation defect, several recombinant versions of BCG have been generated. VPM1002 is a promising recombinant BCG that activates the AIM2 inflammasome and the STING pathway and has also been demonstrated to impart significantly higher protection over conventional BCG in phase I and II trials. ${ }^{12}$ Indeed, VPM1002 is also being trialed in healthcare workers and the elderly to protect against severe COVID-19. While the outcomes of these trials are awaited, based on previous studies, it is relevant and timely to hypothesize that mucosal delivery of BCG combined with a STING pathway activator as an adjuvant may lead to the desired neogenesis of iBALTs in the respiratory mucosa that may offer enhanced or better protection against COVID-19 (figure 1). Most importantly, as the positive impact of $\mathrm{B}$ cells in antitumor immunity is being recognized, specifically those that are associated with TLSs formed post immune checkpoint blockade therapy in sarcoma, melanoma and lung cancer, further research is warranted towards their therapeutic induction across cancers. ${ }^{13}$ Heterologous protection similar to BCG-induced trained immunity has also been reported with respect to measles and polio vaccines. ${ }^{14}{ }^{15}$ Given the critical role of dendritic cells in TLSs and their potent IFN-1 producing characteristic, microbe-based therapeutics, such as oncolytic viruses could also investigate similar immune memory associated effects to increase durable antitumor immune response.
The wide spectrum of protection using BCG vaccine against non-related pathogens and cancer to enhance innate immunity and bridge adaptive immune responses, emphasizes the urgent need to revisit such microbe-based combination therapeutics for cancer treatment, specifically via local administration. Unfortunately, the ongoing global shortage of BCG has affected routine management of patients with bladder cancer in addition to compromising infant vaccination in some countries. The lessons learned from BCG-induced non-specific protection across infectious and non-infectious diseases could be adapted toward development of similar microbe-based therapies such as oncolytic viruses, Bifidobacteria and others that activate IFN-1 via the STING pathway and potentially mediate innate immune sensitization for immune checkpoint blockade via induction of TLS formation.

Contributors MK conceptualized the commentary based on currently reported evidence. AM, CHG and DRS edited and reviewed the commentary.

Funding This work was supported by Queen's University Research Initiation Grant and the Ontario Ministry of Research Innovation and Science, Early Researcher Award to MK.

Competing interests None declared.

Patient consent for publication Not required.

Provenance and peer review Not commissioned; externally peer reviewed.

Open access This is an open access article distributed in accordance with the Creative Commons Attribution Non Commercial (CC BY-NC 4.0) license, which permits others to distribute, remix, adapt, build upon this work non-commercially, and license their derivative works on different terms, provided the original work is properly cited, appropriate credit is given, any changes made indicated, and the use is non-commercial. See http://creativecommons.org/licenses/by-nc/4.0/. 


\section{REFERENCES}

1 Morales A, Eidinger D, Bruce AW. Intracavitary Bacillus CalmetteGuerin in the treatment of superficial bladder tumors. J Urol 1976;116:180-2.

2 Brook B, Harbeson DJ, Shannon CP, et al. Bcg vaccination-induced emergency granulopoiesis provides rapid protection from neonatal sepsis. Sci Trans/ Med 2020;12:pii: eaax4517.

3 Hegarty PK, Sfakianos JP, Giannarini G, et al. COVID-19 and Bacillus Calmette-Guérin: what is the link? Eur Urol Oncol 2020.

4 Hamiel U, Kozer E, Youngster I. SARS-CoV-2 rates in BCGvaccinated and unvaccinated young adults. JAMA 2020. doi:10.1001/jama.2020.8189. [Epub ahead of print: 13 May 2020].

5 O'Neill LAJ, Netea MG. Bcg-Induced trained immunity: can it offer protection against COVID-19? Nat Rev Immunol 2020;20:335-7.

6 Yao Y, Jeyanathan M, Haddadi S, et al. Induction of autonomous memory alveolar macrophages requires $T$ cell help and is critical to trained immunity. Cell 2018;175:e17:1634-50.

7 Phuah JY, Mattila JT, Lin PL, et al. Activated B cells in the granulomas of nonhuman primates infected with Mycobacterium tuberculosis. Am J Pathol 2012;181:508-14.

8 Randall TD, Sanchez A. Anatomical uniqueness of the mucosal immune system (GALT, NALT, iBALT) for the induction and regulation of mucosal immunity and tolerance. 2nd Edn, 2020: 21-4.
9 Jallad S, Goubet S, Symes A, et al. Prognostic value of inflammation or granuloma after intravesival BCG in non-muscle-invasive bladder cancer. BJU Int 2014;113:E22-7.

10 Koti M, Xu AS, Ren KYM, et al. Tertiary lymphoid structures associate with tumour stage in urothelial bladder cancer. Bladder Cancer 2017;3:259-67.

11 Koti M, Chenard S, Nersesian S, et al. Investigating the sting pathway to explain mechanisms of BCG failures in non-muscle invasive bladder cancer: prognostic and therapeutic implications. Bladder Cancer 2019;5:225-34.

12 Nieuwenhuizen NE, Kulkarni PS, Shaligram U, et al. The recombinant Bacille Calmette-Guérin vaccine VPM1002: ready for clinical efficacy testing. Front Immunol 2017;8:1-9.

13 Helmink BA, Reddy SM, Gao J, et al. B cells and tertiary lymphoid structures promote immunotherapy response. Nature 2020;577:549-55.

14 Sørup S, Stensballe LG, Krause TG, et al. Oral polio vaccination and hospital admissions with Non-Polio infections in Denmark: nationwide retrospective cohort study. Open Forum Infect Dis 2016;3:ofv204.

15 Sørup S, Benn CS, Poulsen A, et al. Live vaccine against measles, mumps, and rubella and the risk of hospital admissions for nontargeted infections. JAMA 2014;311:826-35. 